\title{
Chaotic behavior of gas bubble in non-Newtonian fluid: a numerical study
}

\author{
S. Behnia $\cdot$ F. Mobadersani $\cdot$ M. Yahyavi · \\ A. Rezavand
}

Received: 28 December 2012 / Accepted: 18 June 2013 / Published online: 16 July 2013

(C) Springer Science+Business Media Dordrecht 2013

\begin{abstract}
In the present paper, the nonlinear behavior of bubble growth under the excitation of an acoustic pressure pulse in non-Newtonian fluid domain has been investigated. Due to the importance of the bubble in the medical applications such as drug, protein or gene delivery, blood is assumed to be the reference fluid. Effects of viscoelasticity term, Deborah number, amplitude and frequency of the acoustic pulse are studied. We have studied the dynamic behavior of the radial response of bubble using Lyapunov exponent spectra, bifurcation diagrams, time series and phase diagram. A period-doubling bifurcation structure is predicted to occur for certain values of the effects of
\end{abstract}

\footnotetext{
S. Behnia $(\varangle)$

Department of Physics, Urmia University of Technology, Urmia, Iran

e-mail: s.behnia@sci.uut.ac.ir

F. Mobadersani

Department of Mechanical Engineering, Urmia University of Technology, Urmia, Iran

F. Mobadersani

Department of Mechanical engineering, Urmia University, Urmia, Iran

M. Yahyavi

Department of Physics, Bilkent University, 06800 Bilkent, Ankara, Turkey

A. Rezavand

Department of Mechanical engineering, Iran University of Science and Technology, Tehran, Iran
}

parameters. The results show that by increasing the elasticity of the fluid, the growth phenomenon will be unstable. On the other hand, when the frequency of the external pulse increases the bubble growth experiences more stable condition. It is shown that the results are in good agreement with the previous studies.

Keywords Bubble dynamics · Non-Newtonian fluids · Chaotic oscillations · Deborah number . Bifurcation diagrams $\cdot$ Lyapunov spectrum

\section{Introduction}

In view of the escalating use of bubbles in new applications, particularly medical and industrial, research on the growth and collapse of bubbles in different structures and environments has increased [1]. In more important medical applications, bubbles are used for the delivery of drugs [2-4], cancer treatment [5-7], and in the barrier opening of clogged veins and arteries $[8,9]$. In all of these cases, bubbles should move and grow in the blood stream and collapse in the intended location. The researches conducted on blood indicate that considering the blood to be a nonNewtonian fluid correlates well with the experimental results and hence, most of the models presented for fluid field analysis assume the blood to be a nonNewtonian fluid [10-14].

Therefore, the study of bubble growth and its stability in non-Newtonian fluid will be of the most im- 
portant concern [15]. The chaotic behavior of bubbles moving in a non-Newtonian fluid has been investigated experimentally by Jiang et al. [16]. In addition to experimental studies [17-21], there have also been many theoretical investigations on bubble growth [22-26]. In the article presented by Wang et al. [27], the nonlinear vibration of a protein bubble submerged in Bingham liquid has been mathematically modeled, and the bubble's reaction to pressure pulses has been studied. By presenting an analytical model for bubble growth in linear viscoelastic fluids and solving it through the perturbation method, Allen and Roy [28] showed that the increase of Deborah number leads to the increase of bubble oscillation amplitude. In another article, Allen and Roy [29] extended their analytical model to nonlinear nonNewtonian fluid (UCM fluid), and used numerical methods to solve the integro-differential equations. They have also demonstrated the increase of bubble oscillation amplitude with the increase of Deborah number. In the work of Jimenez-Fernandez and Crespo [30], through the development of analytical relations for bubble growth in non-Newtonian fluid field effect by external pulses, the growth of bubbles under the influence of factors like pulse intensity, Reynolds number and the amount of elasticity has been investigated. In this study, it has been emphasized that with the increase of Deborah number, bubble growth will become chaotic and the bubble will approach the state of collapse.

Also, in different theoretical study, the subject of bubble growth in non-Newtonian fluid has showed that in cases where the Reynolds number is of the order 1, the growth and collapse of bubbles can be controlled via Newtonian viscosity. Lind and Phillips [31] have presented the growth of bubbles in non-Newtonian fluids through different constitutive equations. According to their results, at large Deborah numbers, bubble displays a completely elastic behavior and its energy diagram indicates a rebound in bubble growth. Brujan [32] used the perturbation method to study the growth of bubbles in non-Newtonian compressible fluid. He showed that at larger Reynolds numbers, sound emission plays the major role in the damping of bubble oscillations. Also, because of the importance of bubble dynamics, several studies have been conducted on the subject of bubble stability. That is, when the bubble motion gets chaotic, its behavior becomes unpredictable and very hard to deal with $[33,34]$. Hence, the chaotic nature of the equation requires particular tools for resolution, since the analytical and linear solutions are not sufficient.

The main argument of this study is focused on various aspects of the dynamics of bubble in nonNewtonian fluid and, also, the effects of substantial parameters that influence the bubble dynamics are studied in a large domain using chaos theory and considering the measure of the non-Newtonian state of the fluid (Deborah number). Bifurcation and Lyapunov exponent diagrams [35-37] are presented for special cases to determine the chaotic regions. It will represent comprehensive information about extremely nonlinear pulsations of bubble in non-Newtonian fluid at high amplitudes of acoustic pressure where deterministic chaos manifests itself in order to determine the stable regions and chaotic of the system, particularly for drug and gene delivery applications where the applied acoustic pressure is considerably greater than the pressure employed in ultrasound imaging.

\section{Dynamics of spherical bubble in viscoelastic fluids}

The governing equation of bubble growth in nonNewtonian fluid follows the general Rayleigh-Plesset equation (GRP), and with regards to the viscoelastic effects of the fluid, the following integro-differential equation is obtained [29]:

$$
\begin{aligned}
R \ddot{R} & +\frac{3}{2} \dot{R}^{2} \\
& =\frac{1}{\rho}\left[p_{g}-p_{\infty}-\frac{2 \sigma}{R}+2 \int_{R}^{\infty}\left(\frac{\tau_{r r}-\tau_{\theta \theta}}{r}\right) d r\right] .
\end{aligned}
$$

In the above equation, $\tau_{r r}$ and $\tau_{\theta \theta}$ are components of shear stress tensor, which have non-uniform field distribution because of the deformation that exists in the fluid field. Equation (1) has been written for a bubble with radius $R$ which is affected by a pressure field far away from the bubble, $p_{\infty}$, in the form of $p_{0}+$ $P_{a} \sin (\omega t)$, where $p_{0}$ is the ambient pressure. Pressure pulse enters the fluid field with angular frequency $\omega$ and pressure amplitude $P_{a}$. Also, $p_{g}$ and $\sigma$ denote the uniform pressure inside the bubble and surface tension of fluid, respectively. For simplicity, we assume that the internal gas follows a polytropic relationship with exponent $k$, and we have $p_{g}=p_{g_{0}}\left(\frac{R_{0}}{R}\right)^{3 k}$, where $p_{g_{0}}$ 


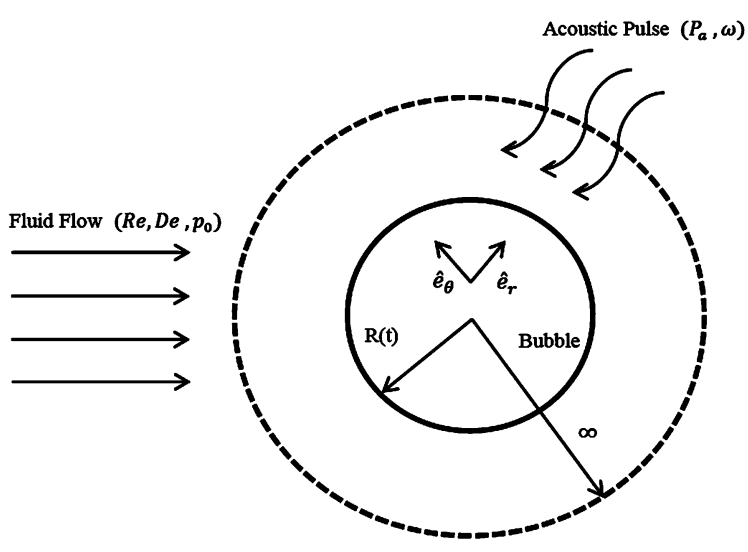

Fig. 1 A single gas bubble immersed in a non-Newtonian fluid

and $R_{0}$ are, respectively, the gas bubble pressure and the bubble radius at the initial equilibrium state. Figure 1 shows a single gas bubble immersed in a NonNewtonian fluid. By considering the upper convective time derivative (UCM) method [28, 29], the radial and theta stress tensor terms will be obtained through the following simplified differential equations:

$$
\left\{\begin{array}{l}
\tau_{r r}+\lambda_{1}\left(\frac{\partial \tau_{r r}}{\partial t}+\frac{R^{2} \dot{R}}{r^{2}} \frac{\partial \tau_{r r}}{\partial r}+\frac{4 R^{2} \dot{R}}{r^{3}} \tau_{r r}\right) \\
=4 \eta_{0} \frac{R^{2} \dot{R}}{r^{3}}, \\
\tau_{\theta \theta}+\lambda_{1}\left(\frac{\partial \tau_{\theta \theta}}{\partial t}+\frac{R^{2} \dot{R}}{r^{2}} \frac{\partial \tau_{\theta \theta}}{\partial r}-\frac{2 R^{2} \dot{R}}{r^{3}} \tau_{\theta \theta}\right) \\
=-2 \eta_{0} \frac{R^{2} \dot{R}}{r^{3}},
\end{array}\right.
$$

where $\eta_{0}$ is the zero shear-rate viscosity, $\lambda_{1}$ is the relaxation time, and $r$ is the distance of each element from the coordinate system's origin. By applying the perturbation method, Allen and Roy [28, 29] solved the above coupled equations and then in 2001, by using the Lagrangian perspective and attaching the coordinates onto the bubble, they have changed variable $y=r^{3}-R^{3}(t)$ and have solved the simplified form of the above equations, with $y=0$ indicating bubble boundary [29]. The upper limit of the integral in Eq. (1) should be selected in such a way that both terms of the shear stress tensor (radial and theta) become zero.

Equation (1) shows the growth of a bubble immersed in a non-Newtonian fluid, which oscillates at its dimensionless radius $R^{*}$ (the $*$ has been omitted in the rest of the article) under the influence of an exter- nal pressure pulse. The Deborah number $\left(D e=\lambda_{1} \omega\right)$ is a dimensionless number which designates the time required for fluid response divided by the time of flow pulse; in fact, it measures the non-Newtonian state of the fluid.

If the following definitions of non-dimensional time, radius, radial spatial variable, stress and Reynolds number are being used [29],

$$
\begin{aligned}
& \bar{t}=\omega t ; \quad \bar{R}=R / R_{0} ; \quad \bar{r}=r / R_{0} ; \\
& \bar{\tau}=\tau \frac{R_{0}}{\eta_{0}} \sqrt{\rho / \rho_{0}} ; \quad \operatorname{Re}=\frac{\rho \omega R_{0}^{2}}{\eta_{0}},
\end{aligned}
$$

then Eq. (1) can be rewritten in non-dimensional form,

$$
\begin{aligned}
R \ddot{R}+\frac{3 \dot{R}^{2}}{2}= & \frac{p_{0}}{\rho \omega^{2} R_{0}^{2}}\left[\left(1+\frac{2 \sigma_{s}}{p_{0} R_{0}}\right)\left(\frac{1}{R}\right)^{3 k}\right. \\
& \left.-\left(\frac{2 \sigma_{s}}{p_{0} R_{0}}\right)\left(\frac{1}{R}\right)-(1+\alpha \sin (t))\right] \\
& +\frac{1}{R e} \frac{1}{\omega R_{0}} \sqrt{\frac{p_{0}}{\rho}} \times \int_{r}^{r_{1}}\left(\frac{\tau_{r r}-\tau_{\theta \theta}}{r}\right) d r,
\end{aligned}
$$

where $\alpha$ is the ratio of the acoustic forcing pressure amplitude to the ambient pressure. In dimensionless form, the stress tensor components of Eq. (2) could be rewritten as

$$
\left\{\begin{aligned}
\tau_{r r} & +D e\left(\frac{\partial \tau_{r r}}{\partial t}+\frac{R^{2} \dot{R}}{r^{2}} \frac{\partial \tau_{r r}}{\partial r}+\frac{4 R^{2} \dot{R}}{r^{3}} \tau_{r r}\right) \\
= & 4\left(\omega R_{0} \sqrt{\frac{\rho}{p_{0}}}\right) \frac{R^{2} \dot{R}}{r^{3}}, \\
\tau_{\theta \theta} & +D e\left(\frac{\partial \tau_{\theta \theta}}{\partial t}+\frac{R^{2} \dot{R}}{r^{2}} \frac{\partial \tau_{\theta \theta}}{\partial r}-\frac{2 R^{2} \dot{R}}{r^{3}} \tau_{\theta \theta}\right) \\
& =-2\left(\omega R_{0} \sqrt{\frac{\rho}{p_{0}}}\right) \frac{R^{2} \dot{R}}{r^{3}} .
\end{aligned}\right.
$$

Since the constitutive equations used are based on the incompressible assumption, radiation damping is not considered.

\section{Analysis tools}

There are several mathematical tools available for quantifying bubble stability ranging, the reasons to use maximum Lyapunov exponents and bifurcation structure in the absence of direct mathematical methods are: 
- The maximum Lyapunov exponents, approximated computationally for a wide range of injection values, clearly indicate the chaotic behavior of bubble dynamics.

- The computationally based bifurcation analysis illustrates that the bubble dynamics transits among different regions such as fixed point, chaotic attractors and intermittent behavior.

\subsection{Computation of Lyapunov exponents}

One of the significant ways studying the behavior of bubble dynamics is to calculate the Lyapunov exponent spectrum which is a measure of the sensitivity of the system to initial conditions and the exponential rates of divergence or convergence of nearby trajectories in state space. The Lyapunov exponents can be considered as "dynamic" measures of attractors complexity and called "time average" [38]. They can be used to characterize chaos and bifurcation which are common as nonlinear effects in bubble dynamics. The Lyapunov exponents are defined as follows.

Consider two nearest neighboring points (usually the nearest) in phase space at time 0 and $t$, with distances of the points in the $i$ th direction $\left\|\delta x_{i}(0)\right\|$ and $\left\|\delta x_{i}(t)\right\|$, respectively. The Lyapunov exponent is then defined through the average growth rate $\lambda_{i}$ of the initial distance,

$$
\begin{aligned}
& \frac{\left\|\delta x_{i}(t)\right\|}{\left\|\delta x_{i}(0)\right\|}=2^{\lambda_{i} t} \quad(t \rightarrow \infty) \quad \text { or } \\
& \lambda_{i}=\lim _{t \rightarrow \infty} \frac{1}{t} \log _{2} \frac{\left\|\delta x_{i}(t)\right\|}{\left\|\delta x_{i}(0)\right\|} .
\end{aligned}
$$

There are three possibilities:

- If $\lambda<0$ the trajectories go close to each other $\rightarrow$ stable radial oscillation.

- If $\lambda=0$ the orbits maintain their relative positions, they are on a stable attractor.

- If $\lambda>0$ implies that the orbit never falls within the basin of attraction of any periodic orbits $\rightarrow$ unstable radial oscillation (chaotic behavior).

The existence of a positive Lyapunov exponent is the indicator of chaos showing neighboring points with infinitesimal differences at the initial state abruptly separate from each other in the $i$ th direction. On the other hand, even if the initial states are near each other, the final states are very different. Hence this phenomenon is sometimes called a sensitive dependence on initial conditions. Commonly, Lyapunov exponents $(\lambda)$ can be extracted by observed signals by the following different methods:

- Based on the opinion of following the time-evolution of nearby points in the state space.

- Based on the estimation of local Jacobi matrices.

The first method is usually called Wolf algorithm [40] and it provides an estimation of the largest Lyapunov exponent only. The second method is capable of estimating all the Lyapunov exponents. Using one of these methods, the Lyapunov exponent is calculated rather than a given control parameter. So, there is a slight increase in value of the control parameter and the Lyapunov exponent is calculated for the new control parameter. By continuing this method the Lyapunov exponent spectrum of the bubble dynamics system is plotted versus the control parameter.

\subsection{Bifurcation diagrams}

Period-doubling, quasi-periodicity and intermittency [41] are well known routes of transition from periodic to chaotic behavior with their origins in local bifurcations. A qualitative change in the dynamical behavior of a system, such as dynamics of bubbles in ultrasonic fields, when a parameter of the system is varied, is called a bifurcation. As it is known, an appropriate method of studying bifurcation is by bifurcation diagram, which provides a helpful insight into the transition between different types of behavior that can occur as one parameter of the system alters.

In this paper, the dynamical behavior of the bubble radial oscillations is studied by plotting the bifurcation diagrams of the normalized radius of the bubble in comparison with different control parameters. The analysis of the bifurcation diagram was carried out in the Poincare section $(P)$. To choose the appropriate Poincaré section, we use the general technique of setting one of the phase space coordinates to zero. In our analysis the following condition was used:

$$
P \equiv \max _{R}\{(R, \dot{R}): \dot{R}=0\}
$$

which gives the maximal radius from each acoustic period. Also, this condition was used to plot the bifurcation diagram of a cavitation bubble in [42]. In order to generate the bifurcation points, the equation of the bubble motion was solved numerically for 900 acoustic cycles of the lower frequency and then a Poincaré 
section was constructed. Considering just the last 300 cycles convinces that the initial transient behavior is eliminated. To create the bifurcation diagram:

- on the $x$-axis is plotted a bifurcation parameter which is varied;

- on the $y$-axis plotted is the asymptotic behavior of a sampled state parameter as a discrete point $\left(\frac{R}{R_{0}}\right)$.

After the system reached its steady state, up to 600 orbits of $\frac{R}{R_{0}}$ in the condition of $\theta_{0}=0$ Poincaré section were plotted in the bifurcation diagram versus bubble control parameter. This method continued through increasing the control parameter and the new resulting discrete points were plotted in the bifurcation diagram versus the new control parameter. For a full discussion on the bifurcation diagram and Lyapunov exponent spectrum, their utilization in order to study the bubble dynamics, one can refer to [43, 44].

\section{Results and discussion}

In this section, we explain the dynamics of bubble in non-Newtonian fluid by using standard methods of nonlinear dynamics and theory of deterministic chaos, because of its importance, the stability of bubbles under the influence of viscoelasticity term, the Deborah number, the amplitude and frequency of the acoustic pulse.

At $t=0$, no pressure pulse is applied to the field and, thus, there is no shear stress distribution, and assuming $R(0)=1$, equations will be solved in the coupled form (see Appendix). In this study, the UCM method has been used, since it is the most appropriate technique for the modeling of bubbles in medical applications [28, 29].

\subsection{Impact of Deborah Number}

Deborah number $(D e)$, as a measure of the nonNewtonian state of the fluid, best describes the bubble stability. By plotting the time series in this study, it has been demonstrated that $D e$ is an important parameter in the nonlinear oscillation of a bubble, and its increase causes the collapse of a bubble. The attractor dimension is an appropriate criterion for measuring the complexity of an attractor in the phase space, and properly delineates the instability threshold of a bubble. It can be stated that with the increase of $D e$ number, bubble growth inside the blood fluid becomes chaotic, and due to instability, its control becomes impossible, so an elasticity threshold should be determined for the fluid. Also, other research works have reported the instability of time series with the increase of $D e$ [22, 24, 28-30,45], which did not determine a threshold of $D e$ for stability of bubble. We examine the stability of bubble growth in non-Newtonian fluid by considering the $D e$ number of the bubble. Radial motion of single bubble dynamics is investigated versus a prominent domain of $D e$ number from 2 to 7 . Figures 2(a)-2(c) shows the bifurcation diagrams and the corresponding Lyapunov spectrum of the bubble radius when $D e$ number of the bubble is taken as the control parameter with the pressure amplitude of $200 \mathrm{kHz}$ for several values of frequency of the bubble which are 3, 4 and $5 \mathrm{MHz}$, whose stable and chaotic pulsations can be observed in respective parts of Figs.2(d)-2(f). The figure shows the chaotic oscillations of bubble by increasing the values of $D e$ and that the bubble demonstrates more chaotic oscillations as the frequency is decreasing.

Figure 3 shows a selection of associated radiustime profiles illustrating the cascade to chaos through a period-doubling bifurcation. As can be seen, the motion is initially stable with period one and undergoes early cascades of period-doubling to chaos. Figure 3(a) shows a stable one-period radius-time profile of bubble at $D e=2.2$, which undergoes a perioddoubling bifurcation. Also, for this value of the applied $D e$, the oscillations settle on one stable limit cycle (Fig. 3(d)). The global dynamics enters a more organized region of period-4 oscillation for $4.25<D e<$ 4.75. In this region the oscillation settles onto four stable limit cycles in the state space trajectory (Fig. 3(e)). Figure 3(c) indicates the chaotic oscillations of a bubble at parameter values $\left(P_{a}=0.2, f=4, D e=6.5\right)$. The orbit projection (Fig. 3(f)) reveals the strange attractor that is created. As the figures show, by increasing number $D e$, the period doubling occurs and at higher $D e$ values, the dynamics of the system become totally chaotic. This value, which differs according to $P_{a}$ and $f$ value of the system, illustrates the stability limit of the system. This effect of $D e$ number has also been stated in other research works by plotting time series [22, 24, 28-30]. Diagrams in Figs. 3(d)3(f) show that at small values of $D e$, Lyapunov exponent is negative, which indicates that with the reduction of the non-Newtonian effects of the fluid, trajec- 
Fig. 2 Bifurcation diagrams and the corresponding Lyapunov spectrum of a bubble with $1 \mu \mathrm{m}$ initial radius versus pressure and Deborah number. A control parameter is Deborah number (2-7) while (a) the driving frequency is $3 \mathrm{MHz}$, (b) the driving frequency is $4 \mathrm{MHz}$, (c) the driving frequency is $5 \mathrm{MHz}$, (d) corresponding Lyapunov spectrum of (a), (e) corresponding Lyapunov spectrum of (b), and (f) corresponding Lyapunov spectrum of (c). All other physical parameters were kept constant at values given in Table 1
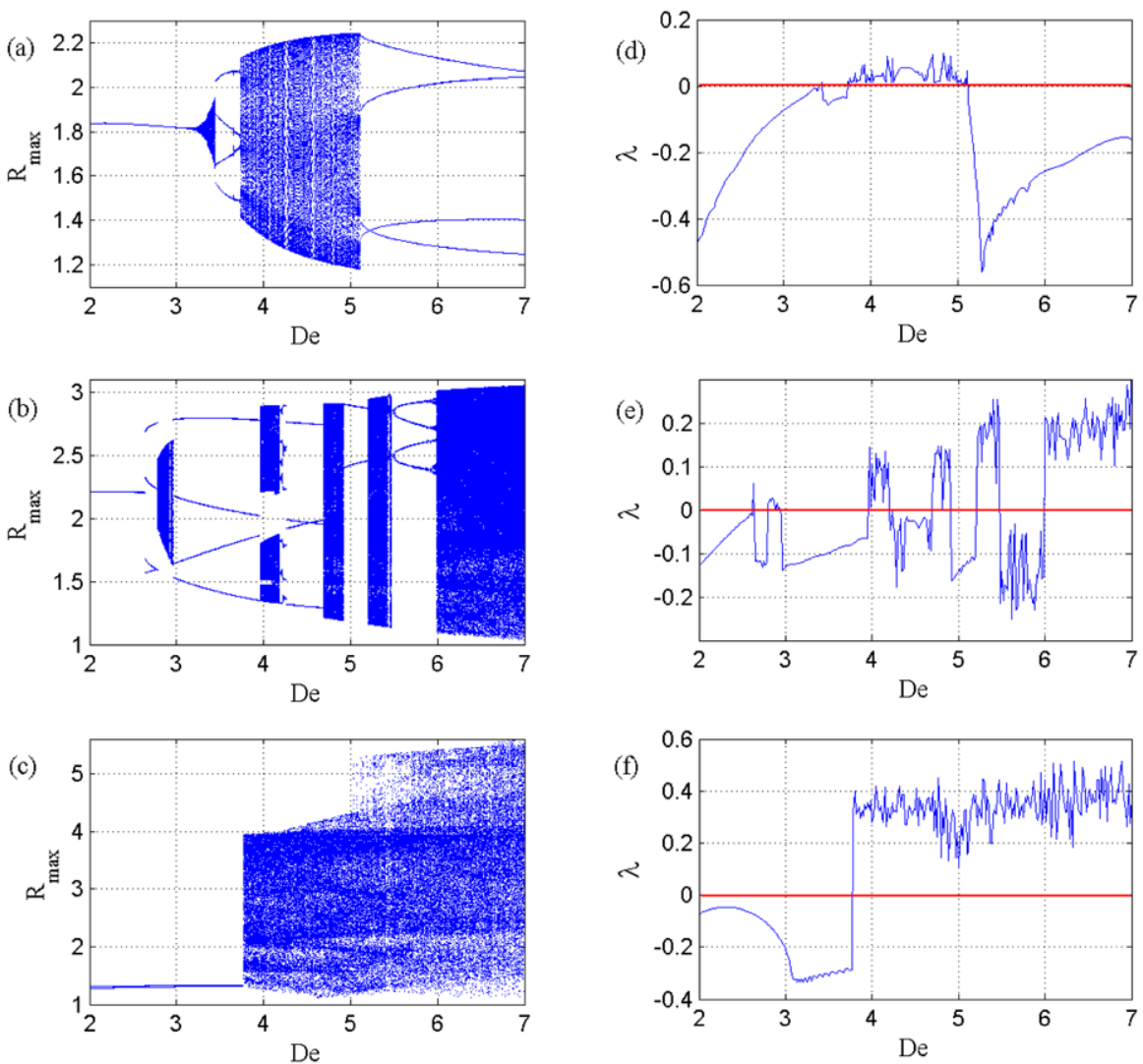
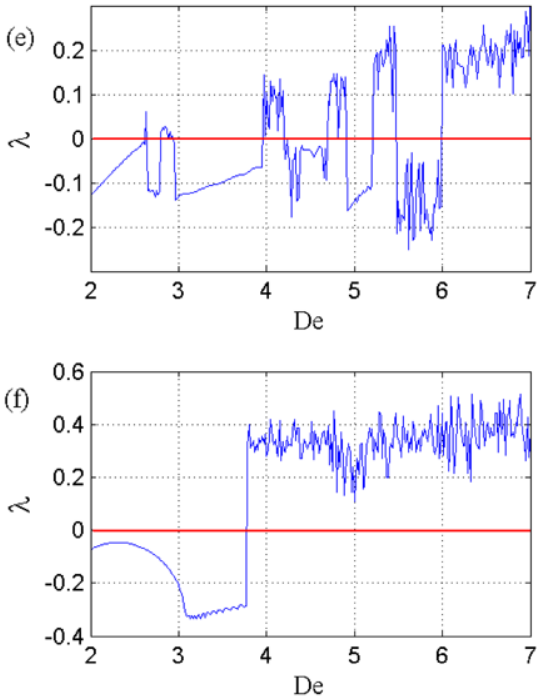

Table 1 Constant parameters used in the general RayleighPlesset equation [28, 29]

\begin{tabular}{llll}
\hline Symbol & Description & Units & Value \\
\hline$\sigma$ & Fluid static surface tension & $\mathrm{dyn} / \mathrm{cm}$ & 72.5 \\
$\rho$ & Fluid density & $\mathrm{kg} / \mathrm{m}^{3}$ & 1000 \\
$p_{0}$ & Ambient pressure & $\mathrm{atm}$ & 1 \\
$R_{0}$ & Equilibrium bubble radius & $\mu \mathrm{m}$ & 1 \\
$R e$ & Reynolds number & & 2.5 \\
$k$ & Polytropic exponent & & 1.4 \\
\hline
\end{tabular}

tories' dependence on initial conditions gets smaller, and as time passes, these trajectories converge to each other. While with the increase of $D e$ number, the divergence of trajectories increases, and in the positive regions of Lyapunov exponent, this divergence increases exponentially.

\subsection{Impact of pressure pulse amplitude}

Pressure pulse amplitude is a measure of the intensity of pulses applied to a bubble in a period. Due to the importance of pulse intensity in medical practice and the fact that these pulses should be applied to bubbles in order to collapse them in the blood stream [1], a proper value should be obtained for pulse intensity by which the range of bubble stability can be determined and controlled. Here, these thresholds will be evaluated with respect to various frequencies and $D e$ numbers, by plotting the bifurcation and Lyapunov exponent diagrams. In Fig. $4, P_{a}$ has been considered as the control parameter and the bifurcation and Lyapunov exponent diagrams have been plotted. The chaotic effect of pressure pulse amplitude on bubble dynamics is very clear. In view of Fig. 4 it can be concluded that at $P_{a}=300 \mathrm{kPa}$, for low values of frequency the oscillations of a bubble is unstable. Bubble growth to the initial radius of $1 \mu \mathrm{m}, R e=2.5, f=3 \mathrm{MHz}$ and $D e=3$ with various acoustic pressure amplitudes models the growth of bubble in blood [28]. In Figs. 4(c) and 4(f) these parameters have been used to model the bubble behavior in blood. Figures 4(c) and 4(f) shows that the growth of the bubble will be unstable if $P_{a}$ reaches $220 \mathrm{kPa}$, which determines a threshold of $P_{a}$. The ef- 
Fig. 3 Time series and trajectory in state space projection plot of a bubble radius driven by $1 \mu \mathrm{m}$ initial radius, $4 \mathrm{MHz}$ of the riving frequency and $200 \mathrm{kPa}$ of pressure while: (a) the Deborah number is 2.2, (b) the Deborah number is 4.5 , (c) the Deborah number is 6.5 , (d) corresponding trajectory in state space projection of (a), (e) corresponding trajectory in state space projection of (b), and (f) corresponding trajectory in state space projection of (c). All other physical parameters were kept constant at values given in Table 1 (a)

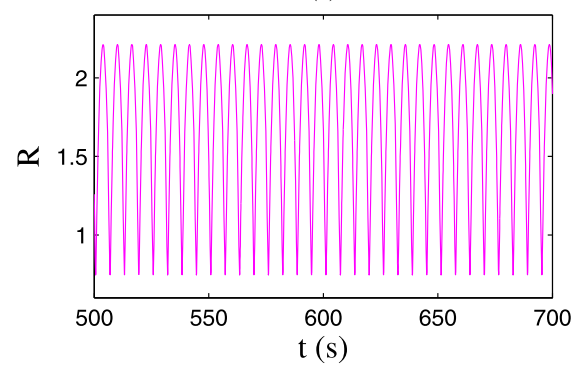

(b)

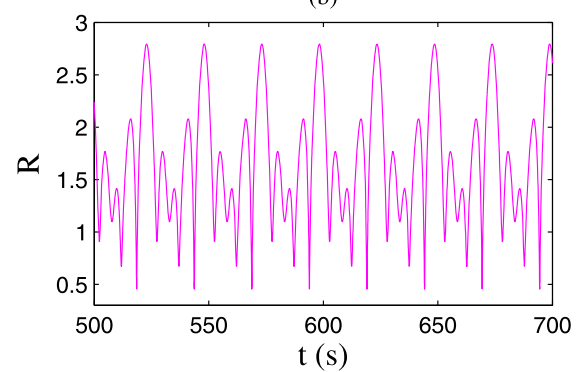

(c)

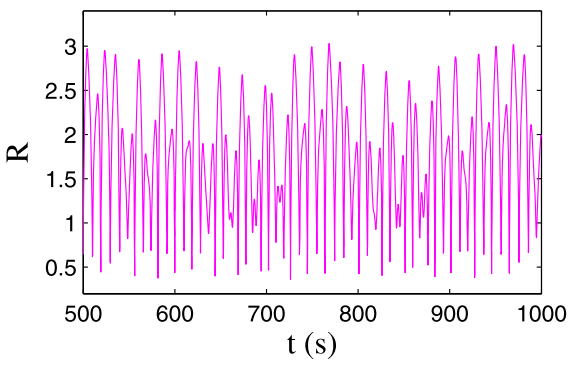

(d)

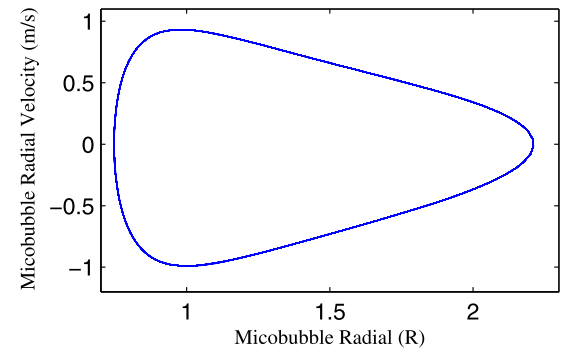

(e)

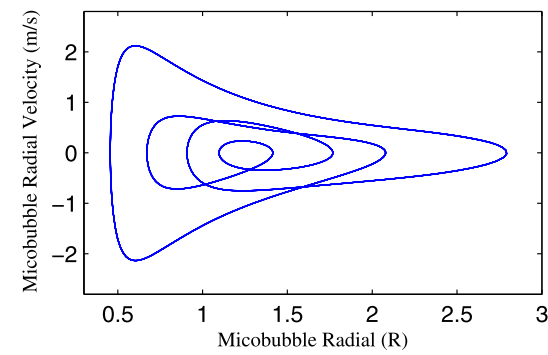

(f)

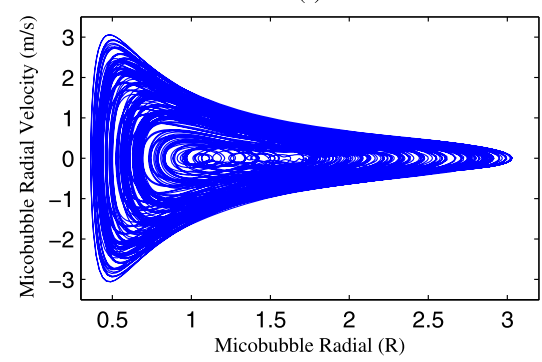

fect of $P_{a}$ as normal stress at high frequencies lead to bubble stability and, thus, the reduction of bubble radius. While with the increase of $P_{a}$ and bubble stability due to normal effects, high amount of stresses will be eliminated. Also, Fig. 4 illustrates the transition through the instability threshold; however, due to the applied frequency being high, this transition occurs at a larger $P_{a}$. The Lyapunov exponent diagram shows that in these conditions, by making the pressure pulse amplitude larger, Lyapunov exponent at $P_{a}=800 \mathrm{kPa}$ will be larger than zero. Comparing the figures presented above, some limits of stable behavior may be determined for the bubble.

Comparison of Figs. 4 and 5 properly illustrates the effect of $P_{a}$ on chaotic degree of the system. Increasing $P_{a}$ means applying higher normal stresses to the surface of the bubble, which will simulate the bubble growth. As Figs. 4 and 5 show, by increasing $P_{a}$, stable range of the bubble decreases dras- tically, and reduces the windows in bifurcation diagram. These results have also been verified in previous works [28-30]. As $P_{a}$ is being increased more, the possibility of the bubble collapse increases. As a result the control of $P_{a}$ should be considered in medical applications. By comparing these figures, it can be found that the oscillation amplitude of bubble radius decreases considerably at high frequencies and low Deborah number, which could be due to the application of large pressure pulses on bubble surface at a shorter time. It can be concluded that the amplitude of the pressure pulse causes the instabilities in the bubble behavior, and this confirms the findings of other studies [28, 29].

\subsection{Impact of pressure pulse frequency (f)}

In order to get more information about the bubble growth in blood (for the purpose of finding periodic or- 
Fig. 4 Bifurcation diagrams and the corresponding Lyapunov spectrum of a bubble with $1 \mu \mathrm{m}$ initial radius and Deborah number is 3 versus pressure and Deborah number, control parameter is pressure while (a) the driving frequency is $7 \mathrm{MHz}$, (b) the driving frequency is $5 \mathrm{MHz}$, (c) the driving frequency is $3 \mathrm{MHz}$, (d) corresponding Lyapunov spectrum of (a), (e) corresponding Lyapunov spectrum of (b), and (f) corresponding Lyapunov spectrum of (c). All other physical parameters were kept constant at values given in Table 1
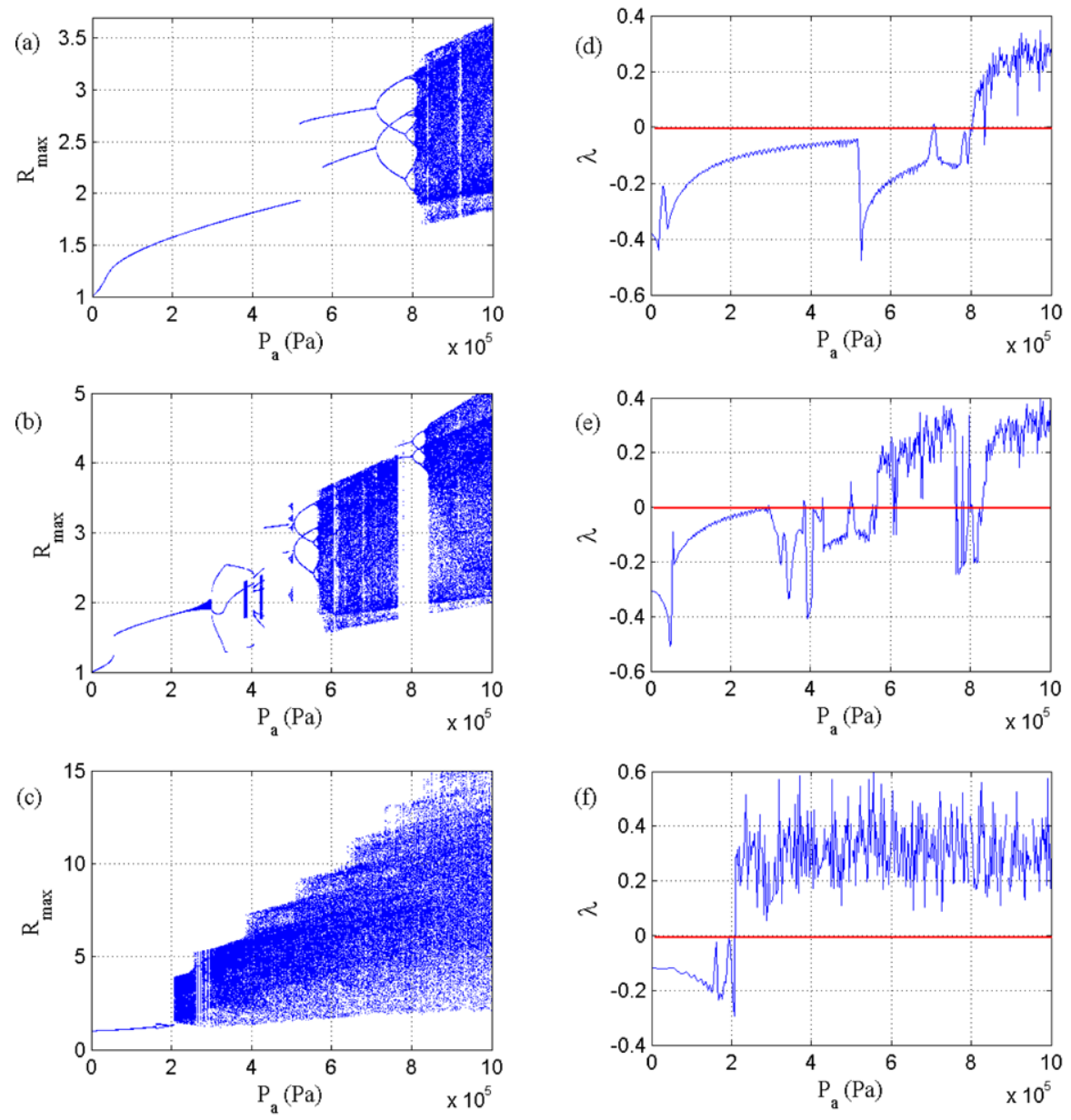

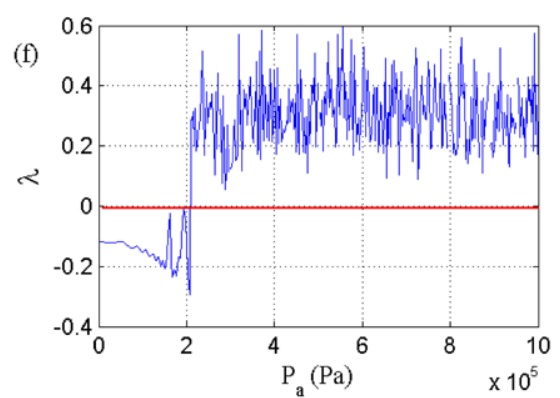

bits and their stability), we calculated numerous bifurcation diagrams of the bubble dynamics considering several values for driving the frequency. In Fig. 6, bifurcation diagrams for the conditions of bubble growth in blood (conditions cited above) have been shown for various pressure pulse amplitudes and $D e$ numbers. The control parameter in the bifurcation diagrams is pressure pulse frequency in order to evaluate the effects of frequency on the stability of bubble at various pressure pulse amplitudes and $D e$ numbers. From these figures it can be concluded that with the increase of the pressure pulse frequency, the bubble becomes more stable and the amplitude of bubble radius decreases considerably. According to the bubble growth equation, the frequency of the acoustic pulse is the main parameter in fluctuations over the bubble interface. Most recently, Dual forcing frequency (through applying a periodic perturba- tion [46]) methods of control have been proven to be successful in the controlling chaotic oscillations of bubble. This method is usually presented a technique based on using periodic perturbation to suppress chaotic oscillations of a spherical cavitation bubble.

It can be understood from the results that the motions of bubble can be chaotic or stable in particular ranges. The results are in agreement with the prior studies clearly highlighting that bubbles are dependent on the driving frequency variations $[22,28,29,46-$ 48]. Most of the results demonstrate the uncontrollable and chaotic motion in a bubble dynamics. In dissimilar situations and values for controlling parameters such as: pressure, frequency and the Deborah number, bubble shows various motions and oscillations by themselves and in addition they change their motion from one type to another. This involves simple pe- 
Fig. 5 Bifurcation diagrams and the corresponding Lyapunov spectrum of a bubble with $1 \mu \mathrm{m}$ initial radius and $6 \mathrm{MHz}$ the driving frequency versus pressure and Deborah number, control parameter is pressure while (a) Deborah number is 3 , (b) Deborah number is 5, (c) Deborah number is 7 ,

(d) corresponding

Lyapunov spectrum of (a), (e) corresponding Lyapunov spectrum of (b), and (f) corresponding Lyapunov spectrum of (c). All other physical parameters were kept constant at values given in Table 1
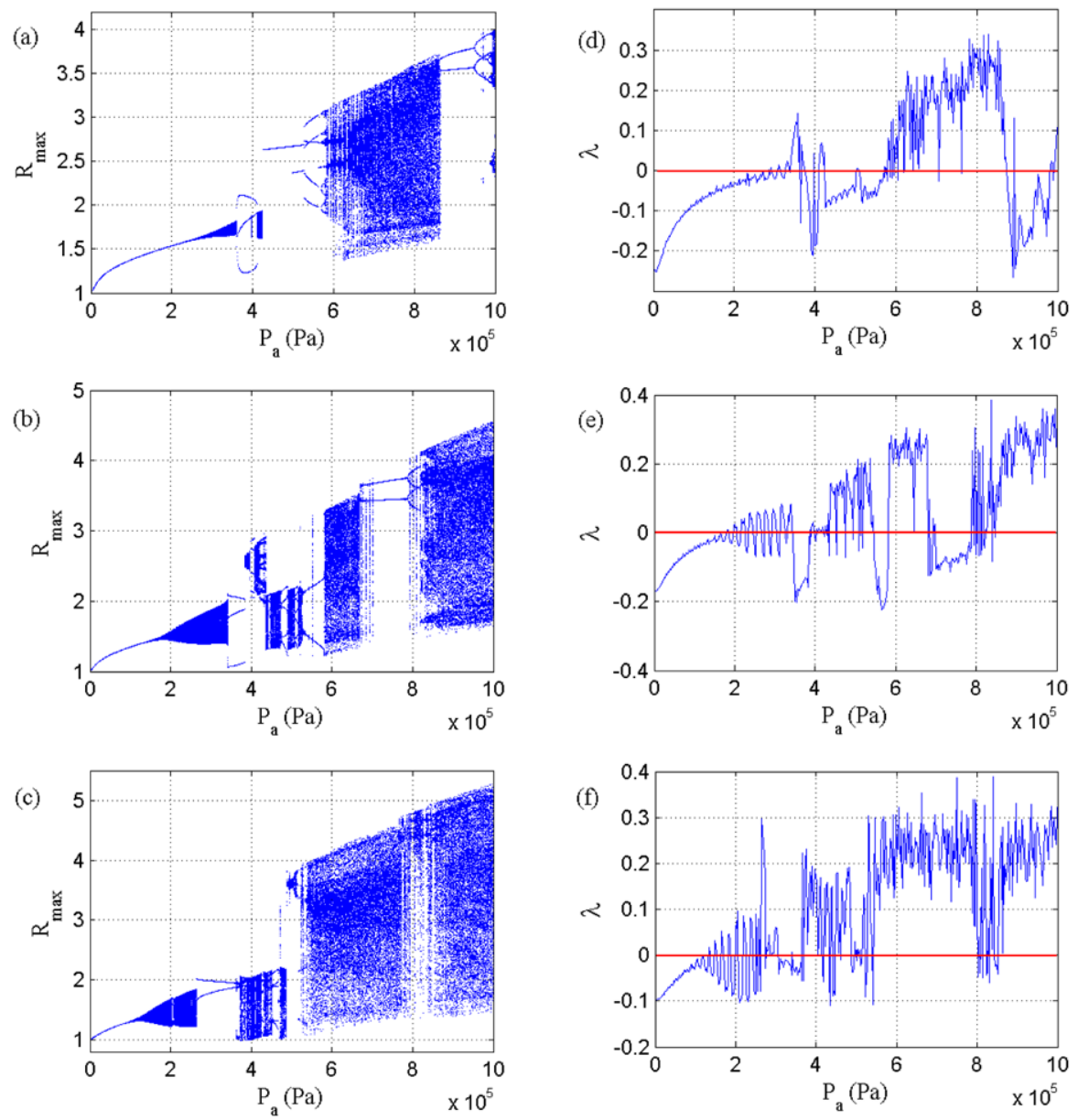

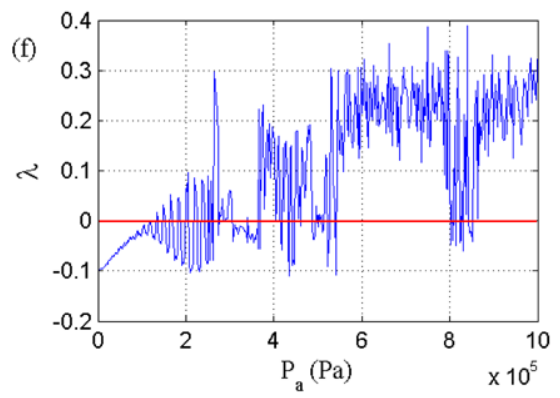

riod one, transformation by period-doubling bifurcation to period two, successive period doubling leading to chaos and high periods, symmetry breaking transition, etc.

\section{Conclusions and outlook}

In this article, bubble stability in non-Newtonian fluid has been investigated through the chaos theory and the ranges in which bubble has stable behavior have been shown by diagrams and also been tabulated to show stability limits of the bubble, which is extremely important in applications. The presented results indicate that the Deborah number, which is a measure of the non-Newtonian state of the fluid, severely affects bubble stability, and with the increase of Deb- orah number, bubble experiences irregular oscillations. These findings confirm the results reported in $[22,24,28-30,45]$. In view of this fact, the injection and conveyance of bubbles in the blood stream should be performed very carefully, and the nonNewtonian state of blood should be tested and measured. Also, according to the presented diagrams, the increase of acoustic pressure amplitude causes instability in bubble boundary and may lead to bubble collapse. This finding has also been pointed out [28-30]. In addition, by increasing the acoustic wave frequency, which indicates the number of pressure pulses in a time unit, the surface of the bubble could be subjected to pressure force, and its irregular oscillations could be avoided. It has been demonstrated in this article that the increase of pressure pulse frequency causes the oscillation amplitude to decrease, and leads to bubble stability. 
Fig. 6 Bifurcation diagrams of a bubble with $1 \mu \mathrm{m}$ initial radius versus pressure, driving frequency and Deborah number, control parameter is and driving frequency while (a) Deborah number is 3 and $P_{a}=200 \mathrm{kPa}$,

(b) Deborah number is 5 and $P_{a}=200 \mathrm{kPa}$,

(c) Deborah number is 7 and $P_{a}=200 \mathrm{kPa}$,

(d) Deborah number is 3 and $P_{a}=400 \mathrm{kPa}$,

(e) Deborah number is 3 and $P_{a}=600 \mathrm{kPa}$,

(f) Deborah number is 3 and $P_{a}=800 \mathrm{kPa}$. All other physical parameters were kept constant at values given in Table 1 (a)

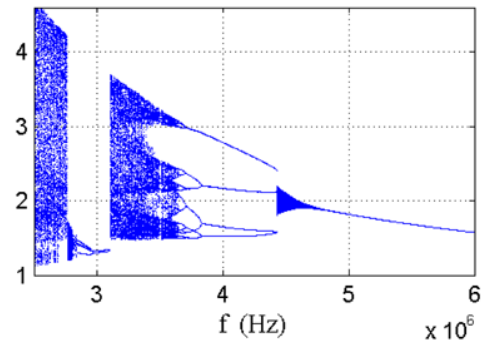

(b)

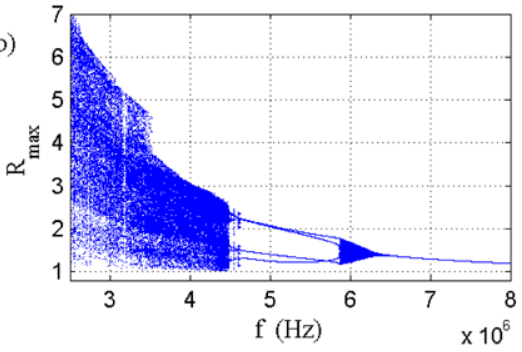

(c)

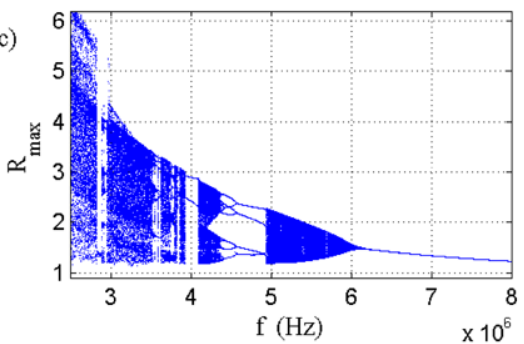

(d)

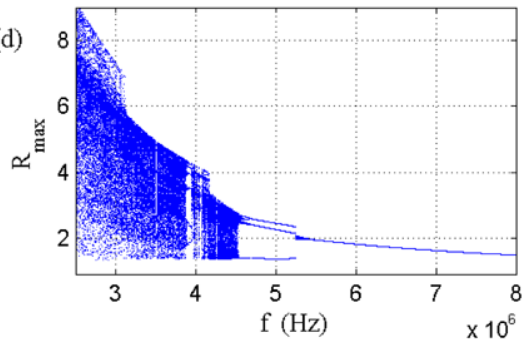

(e)

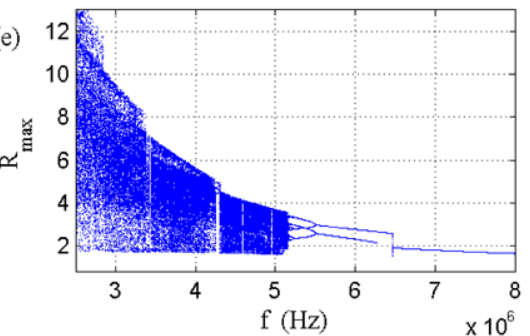

(f)

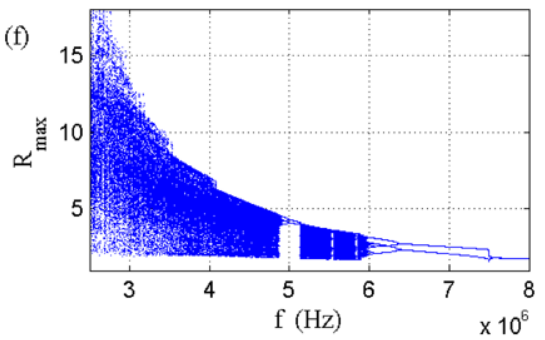

By focusing on the mechanisms governing the transition from the chaotic oscillations to the stable region, this study opens a new horizon in studying chaotic behavior of nonlinear dynamics of gas bubble in nonNewtonian fluid. It is essential to consider the impression of the bubble-bubble interaction in choosing the control parameter, since the bubble pulsation is affected by interacting surrounding bubbles [49]. Based on the results, the global dynamics exhibits complicated behavior that undergoes a series of bifurcations as the pressure amplitude increases. In general, the introduced method can be used for studying the behavior of cluster with large number of bubbles.

\section{Appendix: Stability analysis}

Equations (4) and (5) can be expressed as a system of first-order ordinary differential equations in which the zero point is located on the wall of the spherical bubble:

$$
\left\{\begin{aligned}
\frac{d R}{d t}= & U, \\
\frac{d U}{d t}= & {\left[-\frac{3}{2} U^{2}+\frac{p_{0}}{\rho \omega^{2} R_{0}^{2}}\left((1+W e)\left(\frac{1}{R}\right)^{3 k}\right.\right.} \\
& \left.\left.-W e\left(\frac{1}{R}\right)-(1+\alpha \sin (t))\right)\right] \frac{1}{R} \\
& +\frac{1}{R} \frac{2}{3 R e}\left(\frac{1}{\omega R_{0}} \sqrt{\frac{p_{0}}{\rho}}\right) \\
\times & \int_{0}^{\infty}\left(\frac{\tau_{r r}(y, t)-\tau_{\theta \theta}(y, t)}{y_{i}+R^{3}}\right) d y \\
\frac{d \tau_{r r}(y, t)}{d t}= & \left(\left(\frac{-4 R^{2} \dot{R}}{y_{i}+R^{3}}\right)-\frac{1}{D e}\right) \tau_{r r} \\
& +\frac{4}{D e}\left(\omega R_{0} \sqrt{\frac{p_{0}}{\rho}}\right)\left(\frac{R^{2} \dot{R}}{y_{i}+R^{3}}\right) \\
\frac{d \tau_{\theta \theta}(y, t)}{d t}= & \left(\left(\frac{2 R^{2} \dot{R}}{y_{i}+R^{3}}\right)-\frac{1}{D e}\right) \tau_{r r} \\
& \left.-\frac{\left.2 R_{0} \sqrt{\frac{p_{0}}{\rho}}\right)\left(\frac{R^{2} \dot{R}}{y_{i}+R^{3}}\right)}{D^{2}}\right)
\end{aligned}\right.
$$


We is the Weber number, defined as

$W e=\frac{2 \sigma}{p_{c} R_{0}}$.

Also, in above equation the initial conditions are taken as

$$
\begin{aligned}
& R(0)=1\left[R_{0}\right], \\
& \tau_{\theta \theta}(0)=\tau_{r r}(0)=0, \\
& U(0)=0 .
\end{aligned}
$$

This study is conducted for $D e \sim O(1)$ to avoid numerical difficulties because of the division by this quantity in Eq. (7).

\section{References}

1. Suzuki, R., Takizawa, T., Negishi, Y., Utoguchi, N., Maruyama, K.: Effective gene delivery with novel liposomal bubbles and ultrasonic destruction technology. Int. J. Pharm. 354, 49-55 (2008)

2. Hernot, S., Klibanov, A.L.: Microbubbles in ultrasoundtriggered drug and gene delivery. Adv. Drug Deliv. Rev. 60, 1153-1166 (2008)

3. Ibsen, S., Benchimol, M., Simberg, D., Schutt, C., Steiner, J., Esener, S.: A novel nested liposome drug delivery vehicle capable of ultrasound triggered release of its payload. J. Control. Release 155, 0168 (2011)

4. Husseini, G.A., Diaz de la Rosa, M.A., Richardson, E.S., Christensen, D.A., Pitt, W.G.: The role of cavitation in acoustically activated drug delivery. J. Control. Release 107, 253-261 (2005)

5. Frenkel, V.: Ultrasound mediated delivery of drugs and genes to solid tumors. Adv. Drug Deliv. Rev. 60, 1193$1208(2008)$

6. Hynynen, K.: Ultrasound for drug and gene delivery to the brain. Adv. Drug Deliv. Rev. 60, 1209-1217 (2008)

7. Suzuki, R., Namai, E., Oda, Y., et al.: Cancer gene therapy by IL-12 gene delivery using liposomal bubbles and tumoral ultrasound exposure. J. Control. Release 142, 245$250(2010)$

8. Johnston, B.M., Johnston, P.R., Corney, S., Kilpatrick, D.: Non-Newtonian blood flow in human right coronary arteries: transient simulations. J. Biomech. 39, 1116-1128 (2006)

9. Janela, J., Moura, A., Sequeira, A.: A 3D non-Newtonian fluid-structure interaction model for blood flow in arteries. J. Comput. Appl. Math. 234, 2783-2791 (2010)

10. Razavi, A., Shirani, E., Sadeghi, M.R.: Numerical simulation of blood pulsatile flow in a stenosed carotid artery using different rheological models. J. Biomech. 44, 20212030 (2011)

11. Ashrafizaadeh, M., Bakhshaei, H.: A comparison of nonNewtonian models for lattice Boltzmann blood flow simulations. Comput. Math. Appl. 58, 1045-1054 (2009)
12. Shaw, S., Murthy, P.V.S.N.: Magnetic targeting in the impermeable microvessel with two-phase fluid model nonNewtonian characteristics of blood. Microvasc. Res. 80, 209-220 (2010)

13. Chen, J., Lu, X.-Y.: Numerical investigation of the nonNewtonian blood flow in a bifurcation model with a nonplanar branch. J. Biomech. 37, 1899-1911 (2004)

14. Wang, C., Ho, J.-R.: A lattice Boltzmann approach for the non-Newtonian effect in the blood flow. Comput. Math. Appl. 62, 0898 (2011)

15. Favelukis, M., Albalak, R.J.: Bubble growth in viscous Newtonian and non-Newtonian liquids. Chem. Eng. J. 63, 149-155 (1996)

16. Jiang, S., Ma, Y., Fan, W., Yang, K., Li, H.: Chaotic characteristics of bubbles rising with coalescences in pseudoplastic fluid. Chin. J. Chem. Eng. 18, 18-26 (2010)

17. Schembri, F., Sapuppo, F., Bucolo, M.: Experimental classification of nonlinear dynamics in microfluidic bubbles flow. Nonlinear Dyn. 67, 2807-2819 (2012)

18. Ichihara, M., Ohkunitani, H., Ida, Y., Kameda, M.: Dynamics of bubble oscillation and wave propagation in viscoelastic liquids. J. Volcanol. Geotherm. Res. 129, 37-60 (2004)

19. Fu, T., Ma, Y., Funfschilling, D., Li, H.Z.: Bubble formation in non-Newtonian fluids in a microfluidic T-junction. Chem. Eng. Process. 50, 438-442 (2011)

20. Frank, X., Dietrich, N., Wu, J., Barraud, R., Li, H.Z.: Bubble nucleation and growth in fluids. Chem. Eng. Sci. 62, 7090-7097 (2007)

21. Shaokun, J., Youguang, M., Wenyuan, F., Ke, Y., Huaizhi, L.: Chaotic characteristics of bubbles rising with coalescences in pseudoplastic fluid. Chin. J. Chem. Eng. 18, 18-26 (2010)

22. Kafiabad, H.A., Sadeghy, K.: Chaotic behavior of a single spherical gas bubble surrounded by a Giesekus liquid: a numerical study. J. Non-Newton. Fluid Mech. 165, 800-811 (2010)

23. Li, H.Z., Mouline, Y., Midoux, N.: Modelling the bubble formation dynamics in non-Newtonian fluids. Chem. Eng. Sci. 57, 339-346 (2002)

24. Jiménez-Fernández, J., Crespo, A.: The collapse of gas bubbles and cavities in a viscoelastic fluid. Int. J. Multiph. Flow 32, 1294-1299 (2006)

25. Li, H.Z., Frank, X., Funfschilling, D., Mouline, Y.: Towards the understanding of bubble interactions and coalescence in non-Newtonian fluids: a cognitive approach. Chem. Eng. Sci. 56, 6419-6425 (2001)

26. Bloom, F.: Bubble stability in a class of non-Newtonian fluids with shear dependent viscosities. Int. J. Non-Linear Mech. 37, 527-539 (2002)

27. Wang, H., Jiang, X., Ma, J., Zhang, W.: Vibration of a single protein bubble in Bingham liquid. J. Hydrodyn., Ser. B 21, 658-668 (2009)

28. Allen, J.S., Roy, R.A.: Dynamics of gas bubbles in viscoelastic fluids. I. Linear viscoelasticity. J. Acoust. Soc. Am. 107, 3167-3178 (2000)

29. Allen, J.S., Roy, R.A.: Dynamics of gas bubbles in viscoelastic fluids. II. Non-linear viscoelasticity. J. Acoust. Soc. Am. 108, 1640-1650 (2000)

30. Jiménez-Fernández, J., Crespo, A.: Bubble oscillation and inertial cavitation in viscoelastic fluids. Ultrasonics $\mathbf{4 3}$, 643-651 (2005) 
31. Lind, S.J., Phillips, T.N.: Spherical bubble collapse in viscoelastic fluids. J. Non-Newton. Fluid Mech. 165, 56-64 (2010)

32. Brujan, E.A.: A first-order model for bubble dynamics in a compressible viscoelastic liquid. J. Non-Newton. Fluid Mech. 84, 83-103 (1999)

33. Sorokin, V.S., Blekhman, I.I., Thomsen, J.J.: Motions of elastic solids in fluids under vibration. Nonlinear Dyn. 60, 639-650 (2010)

34. Sorokin, V.S., Blekhman, I.I., Vasilkov, V.B.: Motion of a gas bubble in fluid under vibration. Nonlinear Dyn. 67, 147-158 (2012)

35. Siewe Siewe, M., Yamgou, S.B., Moukam Kakmeni, F.M., Tchawoua, C.: Chaos controlling self-sustained electromechanical seismograph system based on the Melnikov theory. Nonlinear Dyn. 62, 379-389 (2010)

36. Gao, Q., Ma, J.: Chaos and Hopf bifurcation of a finance system. Nonlinear Dyn. 58, 209-216 (2009)

37. Chen, H., Zuo, D., Zhang, Z., Xu, Q.: Bifurcations and chaotic dynamics in suspended cables under simultaneous parametric and external excitations. Nonlinear Dyn. 62, 623-646 (2010)

38. Dorfman, J.R.: An Introduction to Chaos in Nonequilibrium Statistical Mechanics. Cambridge University Press, New York (1999)

39. Ott, E.: Chaos in Dynamical System. Cambridge University Press, New York (2002)

40. Wolf, A., Swift, J.B., Swinney, H.L., Vastano, A.: Determining Lyapunov exponents from a time series. Physica D 16D, 285-317 (1985)
41. Behnia, S., Yahyavi, M.: Characterization of intermittency in hierarchy of chaotic maps with invariant measure. J. Phys. Soc. Jpn. 81, 124008-8 (2012)

42. Simon, G., Cvitanovic, P., Levinsen, M.T., Csabai, I., Horath, A.: Periodic orbit theory applied to a chaotically oscillating gas bubble in water. Nonlinearity 15, 25-43 (2002)

43. Parlitz, U., Englisch, V., Scheffczyk, C., Lauterborn, W.: Bifurcation structure of bubble oscillators. J. Acoust. Soc. Am. 88, 1061-1077 (1990)

44. Lauterborn, W., Parlitz, U.: Methods of chaos physics and their application to acoustics p bifurcation structure of bubble oscillators. J. Acoust. Soc. Am. 84, 1975-1993 (1988)

45. Albernaz, D.L., Cunha, F.R.: Bubble dynamics in a maxwell fluid with extensional viscosity. Mech. Res. Commun. 38, 255-260 (2011)

46. Behnia, S., Jafari Sojahrood, A., Soltanpoor, W., Jahanbakhsh, O.: Suppressing chaotic oscillations of a spherical cavitation bubble through applying a periodic perturbation. Ultrason. Sonochem. 16, 502-511 (2009)

47. Macdonald, C.A., Gomatam, J.: Chaotic dynamics of microbubbles in ultrasonic fields. Proc. - Inst. Mech. Eng., 220, 333-343 (2006)

48. Behnia, S., Jafari, A., Soltanpoor, W., Jahanbakhsh, O.: Nonlinear transitions of a spherical cavitation bubble. Chaos Solitons Fractals 41, 818-828 (2009)

49. Yasui, Y., Iida, K., Tuziuti, T., Kozuka, T., Towata, A.: Strongly interacting bubbles under an ultrasonic horn. Phys. Rev. E, Stat. Nonlinear Soft Matter Phys. 77, 016609016619 (2008) 